\title{
Electroweak phase transition in the singlet-extended SM
}

\author{
Kaori FUYUTO* ${ }^{\dagger}$ \\ Nagoya University \\ E-mail: fuyutodth.phys.nagova-u.ac.ip
}

We revisit the sphaleron decoupling condition in the real singlet-extended standard model (SM) in the light of the $125.5 \mathrm{GeV}$ Higgs boson. The analysis is conducted by using the finite-temperature one-loop effective potential with daisy resummation. It is also studied how large the Higgs couplings can be in the region where the sphaleron decoupling condition is satisfied. The sphaleron decoupling condition becomes more severe by about $20 \%$ and the deviation of the triple Higgs coupling from the SM value gets larger than that based on the conventional criterion.

Flavor Physics \& CP Violation 2015

May 25-29, 2015

Nagoya, Japan

* Speaker.

${ }^{\dagger}$ This talk is based on work in the collaboration with E. Senaha in Ref. [四]. 


\section{Introduction}

Observational facts imply that our Universe is baryon-asymmetric [D]. In order to explain it, while there are many mechanisms, one attractive scenario is electroweak baryogenesis (EWBG) [B]. In EWBG, the baryon asymmetry can be produced via the first-order electroweak phase transition (EWPT). This scenario can be verified by collider experiments since its mechanism is intimately connected to the Higgs physics. While we expect that the Higgs sector will be clarified with better accuracy at the coming Large Hadron Collider (LHC) and International Linear Collider (ILC), these situation motivate us to minimize the theoretical uncertainties in the EWBG calculations.

For the success of EWBG, the strong first-order EWPT must be satisfied. This condition is usually described by $v_{C} / T_{C}>1$, where $T_{C}$ denotes a critical temperature and $v_{C}$ is the Higgs vacuum expectation value (VEV) at $T_{C}$, and we call this criterion the sphaleron decoupling condition. If the sphaleron decoupling condition is satisfied, the baryon numbers successfully survive until today. However, the condition actually depends on the Higgs mass that is functions of model parameters. Therefore, in order to examine the possibility of EWBG, we need to evaluate the sphaleron decoupling condition with a sufficient accuracy in the light of the Higgs boson. Moreover, in Ref. [田], it is suggested that the triple Higgs boson coupling, which can be measured at ILC, could deviate in the parameter regions where the sphaleron decoupling is satisfied. Therefore, we reevaluate the deviation of the triple Higgs boson coupling based on the refined sphaleron decoupling condition.

In this study [四], we focus on the real singlet-extended SM, and discuss the relationship between the Higgs boson couplings and the sphaleron decoupling condition.

\section{The Model}

The most general Higgs potential at the renormalizable level is given by

$$
V_{0}=-\mu_{H}^{2} H^{\dagger} H+\lambda_{H}\left(H^{\dagger} H\right)^{2}+\mu_{H S} H^{\dagger} H S+\frac{\lambda_{H S}}{2} H^{\dagger} H S^{2}+\mu_{S}^{3} S+\frac{m_{S}^{2}}{2} S^{2}+\frac{\mu_{S}^{\prime}}{3} S^{3}+\frac{\lambda_{S}}{4} S^{4},
$$

where $H$ is the $\mathrm{SU}(2)$ doublet Higgs and $\mathrm{S}$ is the real singlet. Since $\mu_{H S}$ and $\lambda_{H S}$ are the couplings between $H$ and $S$, these parameters are expected to be large in order to realize the first-order EWPT. After two scalars get VEVs, they become

$$
H(x)=\left(\begin{array}{c}
G^{+}(x) \\
\frac{1}{\sqrt{2}}\left(v+h(x)+i G^{0}(x)\right)
\end{array}\right), \quad S(x)=v_{S}+s(x) .
$$

The physical components of scalars are defined by rotating the original basis with a mixing $\alpha$

$$
\left(\begin{array}{l}
h \\
s
\end{array}\right)=\left(\begin{array}{cc}
\cos \alpha & -\sin \alpha \\
\sin \alpha & \cos \alpha
\end{array}\right)\left(\begin{array}{l}
H_{1} \\
H_{2}
\end{array}\right)
$$

where $-\pi / 4 \leq \alpha \leq \pi / 4$ and $H_{1}$ represents $125.5 \mathrm{GeV}$ Higgs boson. From the above definition, it is found that the Higgs couplings to gauge bosons and fermions are scaled by $\cos \alpha$. These couplings normalized to the SM values are given by

$$
\kappa_{V}=\frac{g_{H_{1} V V}^{\mathrm{rSM}}}{g_{h V V}^{\mathrm{SM}}}=\cos \alpha, \quad \kappa_{F}=\frac{g_{H_{1} f f}^{\mathrm{rSM}}}{g_{h f f}^{\mathrm{SM}}}=\cos \alpha .
$$


Since $\kappa_{V}$ and $\kappa_{F}$ are same in this model, we denote them as $\kappa$. In the CMS experiment, it is shown that $\kappa_{V} \in[0.88,1.15]$ and $\kappa_{F} \in[0.64,1.16]$.

The triple Higgs boson coupling is given by

$$
\lambda_{H_{1} H_{1} H_{1}}^{\mathrm{rSM}, \text { tree }}=6\left[\lambda_{H} v c_{\alpha}^{3}+\frac{\mu_{H S}}{2} s_{\alpha} c_{\alpha}^{2}+\frac{\lambda_{H S}}{2} s_{\alpha} c_{\alpha}\left(v s_{\alpha}+v_{S} c_{\alpha}\right)+\left(\frac{\mu_{S}^{\prime}}{3}+\lambda_{S} v_{S}\right) s_{\alpha}^{3}\right] .
$$

It is observed that, if $\mu_{H S}$ and $\lambda_{H S}$ are nonzero values, the triple Higgs boson coupling induces the deviation from the SM prediction. We define the deviation of the triple Higgs boson coupling from the SM value,

$$
\Delta \lambda_{H_{1} H_{1} H_{1}}=\frac{\lambda_{H_{1} H_{1} H_{1}}^{\mathrm{rSM}}-\lambda_{H_{1} H_{1} H_{1}}^{\mathrm{SS}}}{\lambda_{H_{1} H_{1} H_{1}}^{\mathrm{SM}}},
$$

where both $\lambda_{H_{1} H_{1} H_{1}}^{\mathrm{rSM}}$ and $\lambda_{H_{1} H_{1} H_{1}}^{\mathrm{SM}}$ are estimated at 1-loop level in our numerical calculation.

\section{Electroweak phase transition}

In EWBG, the sphaleron process plays a role in generating baryon asymmetry of the Universe. If its inverse process frequently occurs, the generated BAU eventually vanishes. Therefore, in order to preserve the BAU until today, the sphaleron process must be decoupled right after the EWPT. This condition is given by

$$
\Gamma_{B}^{(b)}(T) \sim e^{-E_{\mathrm{sph}} / T}<H(T)
$$

where $\Gamma_{B}^{(b)}(T)$ is the baryon number changing rate in the broken phase, and $H(T)$ is the Hubble constant. $E_{\mathrm{sph}}$ is defined as the energy of the sphaleron. The above equation can be translated into

$$
\frac{v(T)}{T}>\frac{g_{2}}{4 \pi \mathscr{E}(T)}\left[42.97-2 \log \left(\frac{T}{100 \mathrm{GeV}}\right)+\cdots\right],
$$

where we use $E_{\mathrm{sph}}=4 \pi v(T) \mathscr{E}(T)$. The above condition is called the sphaleron decoupling condition. We independently estimate the right-hand and left-hand side in Eq. (B.2). Comparing both of them, we found the parameter region that satisfies Eq. (B.2). For these calculations, the finite-temperature one-loop effective potential with the daisy resummation is used.

\section{Numerical Results}

In the tree-level potential, there are 8 parameters as follows:

$$
\mu_{H}^{2}, \quad m_{S}^{2}, \quad \mu_{S}, \quad \mu_{S}^{\prime}, \quad \mu_{H S}, \quad \lambda_{H}, \quad \lambda_{H S}, \quad \lambda_{S} .
$$

In the numerical analysis, we replace them with the following parameters

$$
m_{H_{1}}, \quad m_{H_{2}}, \quad v, \quad v_{S}, \quad \mu_{H S}, \quad \mu_{S}^{\prime}, \quad \mu_{S}, \quad \alpha,
$$

where $v=1 /\left(2^{1 / 4} \sqrt{G_{F}}\right)$ with $G_{F}$ being a Fermi constant and $m_{H_{1}}=125.5 \mathrm{GeV}$. Fig. $\square$ shows the region that satisfies $v_{C} / T_{C}>\zeta_{\text {sph }}$ in the $\left(m_{H_{2}}, \kappa\right)$ plane. In this figure, we set $v_{S}=90 \mathrm{GeV}$, 


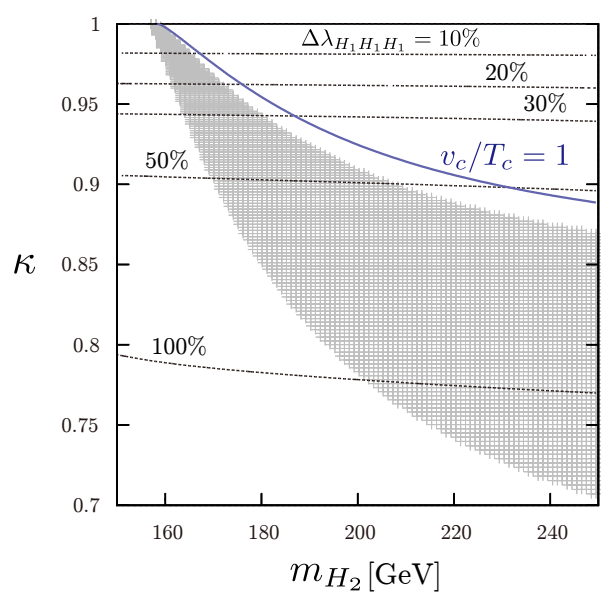

Figure 1: The region where the sphaleron decoupling condition is satisfied in $\left(m_{H_{2}}, \kappa\right)$ plane. The black dotted lines represent the deviation of the triple Higgs boson coupling. The blue line corresponds to the usual criterion, $v_{C} / T_{C}=1$. In this plot, we take $v_{S}=90 \mathrm{GeV}, \mu_{S}^{\prime}=-30 \mathrm{GeV}, \mu_{H S}-80 \mathrm{GeV}$, and $\mu_{S}=0$ $\mathrm{GeV}$.

$\mu_{S}^{\prime}=-30 \mathrm{GeV}, \mu_{H S}-80 \mathrm{GeV}$, and $\mu_{S}=0 \mathrm{GeV}$. The black dotted lines represent $\Delta \lambda_{H_{1} H_{1} H_{1}}=$ $10 \%, 20 \%, 30 \%, 50 \%$ and $100 \%$ from top to bottom. The blue line corresponds to the conventional criterion, $v_{C} / T_{C}=1$. Importantly, the deviation of $\Delta \lambda_{H_{1} H_{1} H_{1}}$ becomes larger than that based on the conventional criterion. Hence, it follows that the mass range of $m_{H_{2}}$ gets narrowed. Its lower bound comes from the condition that the EW vacuum is the global minimum. Although Fig. W shows the wide range of $\kappa$, the LHC constraints allow the only region where $\kappa$ is close to 1 .

\section{Conclusion}

We have improved the sphaleron decoupling condition in the real singlet-extended model in the light of the $125.5 \mathrm{GeV}$ Higgs boson. We analyze it by using the finite temperature one-loop effective potential with thermal resummation. For moderate values of the model parameters, the sphaleron decoupling condition is $v_{C} / T_{C}>1.18$. This value is more severe than the conventional one by about $20 \%$. We also discuss its impacts on the Higgs couplings. As shown in this paper, it is found that the deviation of the triple Higgs boson coupling from the SM value becomes larger than that using the ordinary criterion. Precise measurements of the Higgs boson couplings in the upcoming collider experiments, LHC and ILC may shed light on properties of the EWPT. .

\section{References}

[1] K. Fuyuto and E. Senaha, Phys. Rev. D 90, no. 1, 015015 (2014) [arXiv:1406.0433 [hep-ph]].

[2] P. A. R. Ade et al. [Planck Collaboration], Astron. Astrophys. 571, A16 (2014) [arXiv:1303.5076 [astro-ph.CO]].

[3] V. A. Kuzmin, V. A. Rubakov and M. E. Shaposhnikov, Phys. Lett. B 155 (1985) 36.

[4] S. Kanemura, Y. Okada and E. Senaha, Phys. Lett. B 606, 361 (2005) [hep-ph/0411354]. 